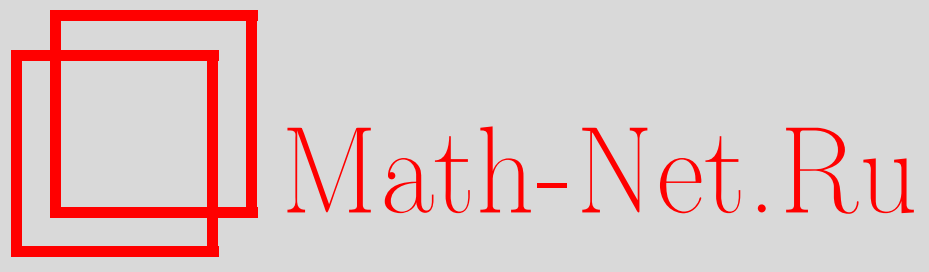

В. Ю. Протасов, Об одном обобщении теоремы Понселе, УМH, 2006, том 61, выпуск 6, 187-188

DOI: https://doi.org/10.4213/rm5583

Использование Общероссийского математического портала Math-Net.Ru подразумевает, что вы прочитали и согласны с пользовательским соглашением http://www . mathnet.ru/rus/agreement

Параметры загрузки:

IP : 54.162 .127 .20

26 апреля 2023 г., 18:16:36

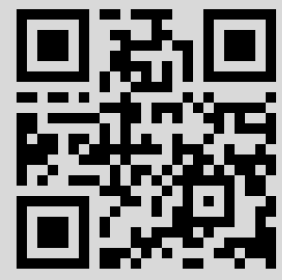




\section{Об одном обобщении теоремы Понселе}

\section{В. Ю. Протасов}

Теоремы о замыкании, такие как теорема Штейнера, теорема Понселе и теорема о зигзаге, изучались во множестве работ и нашли применения в классической геометрии, теории алгебраических кривых, дифференциальных уравнениях и т. д. (см. работы [1]-[4] и библиографию в них). Мы установим, что три классические теоремы о замыкании являются частными случаями более общего утверждения (теорема 1). Везде далее $\mathbb{R}^{2}$ - евклидова плоскость, $(\cdot, \cdot)$ - скалярное произведение.

Процесс Штейнера. Даны окружности $\alpha_{0}$ и $\alpha_{1}\left(\alpha_{1}\right.$ внутри $\left.\alpha_{0}\right)$. Пусть $\mathscr{M}-$ множество окружностей, касающихся $\alpha_{0}, \alpha_{1}$ и лежащих между ними. Для произвольной $\gamma_{1} \in \mathscr{M}$ выбирается $\gamma_{2} \in \mathscr{M}$, касающаяся $\gamma_{1}$, далее для $k \geqslant 3$ окружность $\gamma_{k} \in \mathscr{M}$ касается $\gamma_{k-1}$ и отлична от $\gamma_{k-2}$. Процесс имеет период $n \geqslant 2$, если $\gamma_{n+1}=\gamma_{1}$.

Теорема А (Штейнер). Если процесс Штейнера периодичен для некоторой начальной окружности $\gamma_{1}$, то и для любой $\gamma_{1} \in \mathscr{M}$ он будет иметь тот же период.

Процесс Понселе. Даны окружности $\alpha$ и $\delta$. Через точку $D_{1} \in \delta$ проводится касательная к $\alpha$, она вторично пересекает $\delta$ в точке $D_{2}$. Через $D_{2}$ проводится вторая касательная к $\alpha$, она вторично пересекает $\delta$ в точке $D_{3}$ и т. д. Процесс периодичен, если $D_{n+1}=D_{1}$.

Теорема В (Понселе [5]). Если прочесс Понселе имеет период $n \geqslant 3$, причем точки $D_{1}, \ldots, D_{n}$ различны, то для любой начальной точки $D_{1} \in \delta$, из которой можно провести касательную $\kappa \alpha$, он имеет тот же период.

Общая теорема Понселе верна для пары произвольных невырожденных коник $\alpha$ и $\delta$. Она сводится к случаю двух окружностей проективным преобразованием.

Зигзаг-процесс. Даны окружности $\sigma$ и $\delta$, ни одна из них не содержит центр другой. Дано число $\rho>0$. Возьмем $D_{1} \in \delta, S_{1} \in \sigma$ такие, что $D_{1} S_{1}=\rho$. Далее берем точку $D_{2} \in \delta$, для которой $D_{2} S_{1}=\rho$ и $D_{2} \neq D_{1}$ (если таковой не существует, то $D_{2}=D_{1}$ ), затем точку $S_{2} \in \sigma$ такую, что $S_{2} D_{2}=\rho, S_{2} \neq S_{1}$ (иначе $S_{2}=S_{1}$ ), и т. д. Зигзаг имеет период $n$, если $D_{n+1}=D_{1}$.

Теорема С (теорема о зигзаге [6]). Если зигзаг имеет период $n \geqslant 3$, причем все промежуточных точки различны, то он имеет тот же период для любой точки $D_{1} \in \delta$, из которой можно сделать первый шаг.

Элегантное доказательство теоремы С, использующее дифференциальные уравнения, было получено в [3], первое элементарное доказательство - в [7].

Для окружности $\gamma$, касающейся окружностей $\alpha_{0}, \alpha_{1}$, индекс касания равен 0 , если из двух касаний четное число внутренних, иначе индекс равен 1 . Для $i=0,1 \mathscr{M}_{i}-$ множество окружностей, касающихся $\alpha_{0}$ и $\alpha_{1}$ с индексом $i$. Эти понятия распространяются и на случай, когда окружности вырождаются в прямые или точки.

Обобщенный процесс. На плоскости даны три окружности $\alpha_{0}, \alpha_{1}$ и $\delta$. Каждая из них может вырождаться в прямую или точку. Фиксируем $i \in\{0,1\}$. Предполагаем, что $\delta \notin \mathscr{M}_{i}$. Берется окружность $\gamma_{1} \in \mathscr{M}_{i}$, пересекающая $\delta$ в точках $D_{1}$ и $D_{2}$. Через $D_{2}$ проводим окружность $\gamma_{2} \in \mathscr{M}_{i}$, отличную от $\gamma_{1}$ (если ее не существует, то полагаем $\left.\gamma_{2}=\gamma_{1}\right)$, обозначаем через $D_{3}$ вторую точку пересечения $\gamma_{2}$ и $\delta$, и т. д. Процесс имеет период $n$, если $D_{n+1}=D_{1}$.

Теорема 1. Если обобщенный процесс имеет период $n \geqslant 3$, причем точки $D_{1}, \ldots$, $D_{n}$ различны, то он имеет тот же период для любой окружности $\gamma_{1} \in \mathscr{M}_{i}$, пересекающей $\delta$.

Работа выполнена при поддержке гранта РФФИ 05-01-00066 и гранта НШ-5813.2006.1. 
Теорема $1 \Rightarrow$ теорема А. Все окружности $\gamma_{k}$ принадлежат семейству $\mathscr{M}_{1}$. Тогда теорема А является частным случаем теоремы 1 , в котором в качестве $\delta$ взята окружность с центром $K_{1}$, ортогональная всем окружностям из $\mathscr{M}_{1}$.

Теорема $1 \Rightarrow$ теорема В. Другой частный случай теоремы 1: окружность $\alpha_{1}$ вырождается в точку. После инверсии с центром в точке $\alpha_{1}$ получаем теорему В.

Теорема $1 \Rightarrow$ теорема С. Еще один частный случай теоремы 1: окружности $\alpha_{0}$ и $\alpha_{1}$ концентричны. Взяв в качестве $\alpha_{0}, \alpha_{1}$ окружности, концентрические $\sigma$ и касающиеся всех окружностей с центрами на $\sigma$ и с радиусами $\rho$, получаем теорему С.

Итак, в случае концентрических окружностей $\alpha_{0}, \alpha_{1}$ теорема 1 превращается в теорему о зигзаге; в случае, когда $\alpha_{0}$ является бесконечно удаленной точкой, получаем теорему Понселе; наконец, если $\delta$ является окружностью, ортогональной всем окружностям семейства $\mathscr{M}_{1}$, получаем теорему Штейнера.

Для любой пары окружностей, имеющих разные центры и радиусы, рассмотрим точки $K_{0}, K_{1}$ - центры гомотетий, переводящих одну окружность в другую. Для каждого $i=0,1$ точка $K_{i}$ имеет одну и ту же степень $p_{i}=d^{2}-r^{2}$ относительно всех окружностей $\gamma \in \mathscr{M}_{i}\left(d-\right.$ расстояние от $K_{i}$ до центра $\gamma, r$ - радиус $\left.\gamma\right)$. Окружность с центром $K_{i}$ и радиусом $\sqrt{p_{i}}$ ортогональна всем окружностям семейства $\mathscr{M}_{i}$ и содержит все точки их касания друг с другом.

ДокАзАТЕЛЬСтво теОРЕмЫ 1. Можно считать, что хотя бы две из трех окружностей $\alpha_{0}, \alpha_{1}, \delta$ не вырождаются в точки, иначе утверждение тривиально. Проведя подходящую инверсию, считаем, что эти окружности не обращаются в прямые и имеют разные центры и радиусы. Пусть $i=0$, в случае $i=1$ все аналогично. Докажем, что общие хорды $\delta$ со всевозможными окружностями $\gamma \in \mathscr{M}_{0}$ касаются фиксированной коники $\alpha$. Тогда теорема 1 будет следовать из теоремы Понселе, примененной к конике $\alpha$ и окружности $\delta$. Пусть $O$ - центр $\delta, R$ - ее радиус. Возьмем произвольную окружность $\gamma \in \mathscr{M}_{0}$, пересекающую $\delta$. Пусть $G, r$ - центр и радиус $\gamma$, прямая $l-$ общая хорда $\delta$ и $\gamma$. Положим $a=\overrightarrow{O K_{0}}, b=\overrightarrow{O G}$. Имеем $(a-b)^{2}-r^{2}=p_{0}$. Для произвольной точки $L \in l$ положим $x=\overrightarrow{K_{0} L}$. Точка $L$ имеет равные степени относительно $\delta$ и $\gamma$, откуда $(a+x)^{2}-R^{2}=(a+x-b)^{2}-r^{2}$. Подставляя $r^{2}=(a-b)^{2}-p_{0}$, получаем $2(x, b)=R^{2}-a^{2}+p_{0}$. Так как $p=a^{2}-R^{2}-$ степень $K_{0}$ относительно $\delta$, то $(x, b)=\frac{1}{2}\left(p_{0}-p\right)$. Поскольку центры $G$ всевозможных окружностей $\gamma \in \mathscr{M}_{0}$ описывают конику $\Gamma$ с фокусами в центрах $\alpha_{0}, \alpha_{1}$, прямые $l=\left\{a+x \mid(x, b)=\frac{1}{2}\left(p_{0}-p\right)\right\}$ для всевозможных $b \in \Gamma$ описывают огибающую коники $\alpha$, которая получается из двойственной коники $\Gamma^{*}$ умножением на скаляр $\left(p_{0}-p\right) / 2$ и переносом на вектор $b$.

ЗАмечание 1. Явный вид коники $\alpha$, найденный в доказательстве, позволяет применить теорему Кэли [1] и получить аналитический критерий n-периодичности обобщенного прочесса.

\section{Список литературы}

[1] P. Griffiths, J. Harris, Enseigm. Math. (2), 24 (1978), 31-40. [2] W. Barth, J. Michel, Math. Ann., 295:1 (1993), 25-49. [3] W. L. Black, H. C. Howland, B. Howland, Amer. Math. Monthly, 81:7 (1974), 754-757. [4] V. Burskii, A. Zhedanov, RIAM Symposium № 16ME-S1 (Japan, 2004), article № 24; http://www.riam.kyushu-u.ac.jp/fluid/meeting/16ME-S1. [5] J.V. Poncelet, Traité des properiétés projectives des figures, Bachelier, Paris, 1822 (1st ed.), 1865. [6] O. Bottema, Elem. Math., 20:1 (1965), 1-7. [7] A. Hraskó, Elem. Math., 55:2 (2000), 45-62.

В. Ю. Протасов (V. Yu. Protasov)

Московский государственный университет им. М. В. Ломоносова

E-mail: vladimir_protassov@yahoo.com
Представлено В. М. Тихомировым Принято редколлегией 27.10 .2006 